\title{
EL ANÁLISIS CIENTÍFICO Y EL COMERCIO DEL NEOLÍTICO EUROPEO
}

\section{SCIENTIFIC ANALYSIS AND NEOLITHIC TRADE IN EUROPE}

por

PATRICIA PHILLIPS*

RESUMEN Este pequeño trabajo da cuenta de la situación actual en que se hallan los estudios sobre los intercambios comerciales en la Europa neolítica. Igualmente, describe los principales métodos y las más importantes vías de acceso que los investigadores han usado para abordar el problema.

ABSTRACT This short article discusses recent work on the exchange or trade of artefacts in inorganic raw materials during the European Neolithic. Researchers have used a range of scientific techniques, described in the article, to study different aspects of Neolithic economies.

\section{INTRODUCCIÓN}

El Neolítico europeo llena en dataciones radiocarbónicas el periodo comprendido entre 6000-2500 a.C., que en fechas calibradas abarca desde el 7000 al 3000 A.C. Las cifras más tempranas se refieren a los países mediterráneos y del Sureste europeo, y las más recientes a las zonas del Norte y del Oeste.

Debido a las diferencias cronológicas que acompañaron a los orígenes de la agricultura en Europa, parte del comercio se llevó a cabo entre agricultores y predadores, como sucedió en el caso de la adquisición de hachas pulimentadas por los forrajeros polacos o la de cerámicas por los cazadores italianos. Pero este artículo se centrará sólo en los intercambios realizados entre diferentes grupos de agricultores, asumiendo además que la mayoría de la producción neolítica se usaba en el marco familiar o en el del grupo local.

Gracias a los antropólogos contamos con muchos modelos de comercio o intercambio entre las sociedades primitivas. Por ejemplo, algunas zonas de la Isla de Nueva Guinea solamente han sido visitada por exploradores y antropólogos entre 1920 y 1960, por lo que contamos con excelente documentación sobre el tipo de comercio practicado (Hughes, 1977; Harding, 1967). Parece que era corriente el trueque de objetos con valor ritual, como brazaletes y collares de concha, así como el intercambio de comida, plumas, hachas o cerámica.

\footnotetext{
* Universidad de Sheffield.
} 
Marcel Mauss, en su famoso libro Le Don (1925), habló de los diversos tipos de intercambio primitivos con base en sus análisis de la amplia evidencia antropológica de su época. Creía en el hecho de que comunidades agrícolas primitivas podrían acumular gran cantidad de excedentes que emplearían, por un lado, en intercambios recíprocos basados en obligaciones familiares y, por otro, en el trueque con otros comerciantes de otras tierras. Mauss hablaba del comercio como "un estado inestable entre fiesta y guerra" (1925: 82).

Debido a la desaparición de todo tipo de comida, incluida la sal, solamente podemos seguir la pista de los artículos hechos de materia prima inorgánica. Las principales fuentes de estudio son la cerámica, las conchas horadadas o grabadas y la piedra tallada y/o pulimentada.

Para el estudio del Neolítico inicial y medio, la mayoría de los investigadores enfatizan las largas distancias sobre las que se desarrolla el comercio, así como las actividades especializadas que éste implica. Cuestionan la estabilidad de los contactos comerciales y la posible irregularidad del abastecimiento, y si los primeros no responderían más a las necesidades del productor que a las del consumidor (Bogucki, 1988; Binder y Perlès, 1990). Durante el Neolítico final y el Eneolítico (4000-2500 A.C. en las diferentes zonas), las comunidades agrícolas, entonces implantadas en casi toda Europa, gozaban de un mayor desarrollo de productos locales y regionales, como demuestra la extracción de piedra y mineral llevada a cabo en las minas.

En relación a las rutas comerciales, los arqueólogos han mostrado posibles vías marítimas a lo largo de costas e islas.

Para el Mediterráneo, Johnstone (1980) sugiere que posiblemente se emplearían embarcaciones hechas de haces de juncos. En el Mar del Norte y en el Báltico éstas podrían estar hechas de troncos o de pieles de animales (Case, 1969). Los caminos terrestres junto a los ríos, por valles y pasos de montañas se basan en la distribución de artefactos y en las alineaciones de túmulos. Por otro lado, se han encontrado senderos de madera (Sweet Track, Somerset, en el Suroeste de Inglaterra; Holanda, etc.), indicando las conexiones entre pueblos o aldeas (por ejemplo, Coles y Coles, 1991).

\section{HISTORIA DE LAS INVESTIGACIONES}

La figura 1 muestra brevemente el desarrollo del interés por el estudio del comercio neolítico, sobre todo en las décadas de los setenta y ochenta. Desde los principios de la investigación arqueológica en Europa algunos materiales parecían ser el resultado del intercambio, como es el caso de las conchas marinas descubiertas en yacimientos del interior (Willms, 1982). También las hachas de piedra localizadas en lugares carentes de afloraciones naturales de esas rocas sin duda fueron transportadas allí por grupos humanos. Las primeras investigaciones utilizaban la petrología para detectar los lugares de origen de algunas hachas y cerámicas (Brothwell y Higgs, 1963). Los avances más importantes se llevaron a cabo a mediados de los años sesenta, cuando físicos y químicos desarrollaron numerosas técnicas útiles para aplicaciones arqueológicas. Estas técnicas se usaron para el estudio de piedra (sílex, obsidiana, etc.), cerámica y concha. Los resultados de dichos estudios se dieron a conocer en las conferencias de Arqueometría y en las publicaciones especializadas en la materia. A finales de los años setenta comenzaron a celebrarse reuniones en torno al análisis del sílex (por ejemplo en Madrid en 1991), y más recientemente congresos dedicados a los objetos de piedra pulimentada y las materias primas empleadas en su fabricación.

Los principales propósitos de estos análisis científicos son: 1) la "caracterización" de las fuentes de materia prima utilizadas en el período neolítico, 2) la identificación de las etapas de producción y 3) de las límites de distribución de herramientas, recipientes y adornos de materias primas especiales. Pero los prehistoriadores se interesan también en cómo se obtenía y modificaba la materia prima, en los contextos sociales de la captación y del comercio de la misma, y en el papel social y funcional de los artículos comercializados. 
Fig. 1 CRONOLOGÍA ESQUEMATICA DE LAS INVESTIGACIONES CIENTÍFICAS

\begin{tabular}{|c|c|c|c|}
\hline $1930-39$ & $1940-59$ & $1960-69$ & $1970-89$ \\
\hline \multicolumn{4}{|l|}{$\begin{array}{l}\text { hachas de } \\
\text { piedra }\end{array}$} \\
\hline & & $\begin{array}{l}\text { sílex de minas } \\
\text { obsidiana }\end{array}$ & \\
\hline & & ámbar & $\begin{array}{l}\text { cerámica } \\
\text { variscita } \\
\text { jadeíta } \\
\text { molederas } \\
\text { útiles de sílex } \\
\text { brazaletes de concha }\end{array}$ \\
\hline
\end{tabular}

\section{RESULTADOS GENERALES DE LAS INVESTIGACIONES}

Los estudios sobre la obtención y modificación de la materia prima se han centrado principalmente en los materiales pétreos, concretamente en canteras, minas y talleres. Basten como ejemplos las minas de sílex, desde Polonia a Grime's Graves (Inglaterra); las afloraciones de lava volcánica en el Noroeste de Inglaterra; las canteras de obsidiana de la isla egea de Melos; los talleres de sílex de Andalucía (Sieveking y Newcomer, 1989; Bradley y Edmonds 1993; Torrence, 1986; Ramos Muñoz, e.p.; Vallespi y otros, 1988). En los años sesenta y setenta, los análisis físicos y químicos distinguieron muy bien las diferentes fuentes de obsidiana, pero fue más problemática la distinción de los diversos tipos de sílex (Cowell y Bowman, 1985). Recientemente se ha llevado a cabo un proyecto en la Universidad de Sheffield utilizando la petrología y la micropaleontología para diferenciar los diferentes recursos de sílex del Norte de Inglaterra (Brooks, 1989). Investigaciones parecidas se realizan en el Suroeste de Francia (Masson, 1984) y con el sílex de la Subbética andaluza (Ramos Millán, 1991). La petrología, utilizando las finas láminas de material, es un método muy útil para el ánálisis de muchas materias pétreas y para la cerámica.

Con respecto a la cerámica neolítica, la petrología es probablemente el mejor método de estudio para averiguar sus áreas de fabricación, ya que la mayoría contienen desgrasantes naturales o añadidos. La microscopía de barrido electrónico es buena para indicar la composición y también las temperaturas de cocción. Es posible que, en el futuro, la espectroscopia de plasma inductivamente acoplado, que trabaja con cientos de muestras al mismo tiempo, pueda utilizarse más para el análisis de cerámica fina y de la piedra, incluido el sílex.

\section{CASOS ESTUDIADOS}

El análisis del comercio en el Neolítico europeo se ha llevado a cabo de tres maneras: mediante el estudio de una materia prima única, mediante un solo tipo de artefacto hecho con materias primas diferentes, $y$, por último, a través del estudio de los sistemas de comercio y abastecimiento.

\subsection{Estudio de una materia prima única}

Este tipo de análisis se ha aplicado en casos como el de la variscita, tan utilizada para la fabricación de 
cuentas durante el Neolítico en Iberia (Villalba y otros, 1989). Otro ejemplo es el ámbar utilizado para adornos y objetos rituales en los países bálticos. Las fuentes italianas/sicilianas parecen no ser utilizadas (Beck y Shennan, 1991). La obsidiana es el material que se ha estudiado más a fondo. Tiene la ventaja de que solamente se localiza en unos cuantos lugares, donde el fluído volcanico se ha endurecido convirtiéndose en una extraordinaria sustancia vítrea. Dichas obsidianas varían en su composición, sobre todo en la presencia diferencial de elementos traza. La identificación se hace con el análisis de activación de neutrones y por difracción de rayos X. La mayoría de los recursos europeos de este cristal volcánico se encuentran localizados en islas, excepto en el caso del foco existente en los Cárpatos. Durante el Neolítico, Melos aprovisionaba principalmente a Creta, a las otras islas cicládicas y a la Grecia continental (Renfrew y Aspinall, 1990). La obsidiana de Melos se usaba en la cueva Franchthi antes del Neolítico. Hay que tener en cuenta que las Cícladas se vieron afectadas por el aumento del nivel del mar tras el periodo postglacial, por lo que durante el Neolítico inicial las islas eran mayores y la intervisibilidad más facil. A persar de las prospecciones realizadas, no se han encontrado yacimientos antes del Neolítico final en Melos, sólo unos núcleos de hojas; de ahí que parezca lógico pensar que la deshabitada isla era utilizada tan sólo como fuente de obsidiana. Catherine Perlès (1990a y 1990b), que ha realizado un estudio intensivo de artefactos de obsidiana procedentes de la cueva Franchthi y de otros yacimientos en Grecia continental, indica que durante el Neolítico inicial marineros especializados obtendrían la materia prima en bloques informes o núcleos preformados, y que comerciarían con ellos en las zonas costeras de Grecia continental y en Creta. Durante el Neolítico final, las islas se ocuparían y los grupos cercanos obtendrían sus materias primas directamente mediante lo que Renfrew (1984) ha enominado "comercio independiente".

Cuatro islas del Oeste del Mediterráneo competían como abastecedoras de los territorios cercanos tanto en tierra firme como en las islas. El análisis por activación de neutrones y por difracción de rayos $\mathrm{X}$ ha demostrado el uso durante el Neolítico inicial de la obsidiana de las Lípari y de Pantelleria en el Norte de Sicilia (Francaviglia y Piperno, 1987) y en Malta. En el Noroeste del Mediterráneo, estos análisis muestran la utilización de tres fuentes de obsidiana: las Lípari, Cerdeña y Palmarola (Williams Thorpe y otros, 1984). La mejor obsidiana de Cerdeña, la de tipo A, compitió con éxito con la de las Lípari en el abastecimiento del Norte de Italia y del Sur de Francia durante el quinto milenio a.C. (Phillips, 1986). Con respecto a España, se ha encontrado un núcleo todavía sin analizar en Sabadell (Cataluña). La ausencia de obsidiana en la mayor parte de España en los milenios sexto y quinto a.C. enfatiza el desarrollo distinto peninsular en el Neolítico.

\subsection{Estudio de un solo artefacto hecho de diferentes tipos de materia prima}

Durante muchas decadas se han analizado así los tipos de materias primas utilizados en la fabricación de las hachas en Gran Bretaña. Excepto para las hachas de jadeíta (Woolley, 1983), la petrología ha sido utilizada como principal método de análisis. La mayoría de los sitios de procedencia de las materias primas líticas de Gran Bretaña -más de treinta- se localizan en el Oeste y en el Norte de la isla principal y al Noroeste de Irlanda (Clough y Cummins, 1979 y 1988). Las hachas de Irlanda y de Bretaña se exportaron a Ingalterra y a Escocia. Se han estudiado las canteras y talleres, como en el caso de la toba de Great Langdale (Bradley y Edwards, 1993), y también las rutas de dispersión de las hachas (por ejemplo el valle de Airedale). Las hachas completas y fragmentadas se encuentran tanto en asentamientos como en lugares de rituales. Parecidas investigaciones se están llevando a cabo ahora en toda Europa.

Existe otro artefacto de uso muy extendido hecho en múltiples materias primas en el Neolítico europeo, pero que sin embargo sólo se ha estudiado parcialmente: el brazalete. Los brazaletes de concha o piedra, y aun 
de cerámica en algunas ocasiones, gozaron de gran popularidad en el Neolítico europeo, incluyendo por supuesto el español. En algunos yacimientos existe evidencia de manufactura de brazaletes de piedra (de caliza en la cueva de Fontbrégoua, en Francia, por ejemplo) o de concha (del spondylus gaederopus en Dímini, Grecia). La distribución regional de diferentes tipologías y materias pétreas sugiere un intercambio de brazaletes de piedra más local que el de los brazaletes de concha. No obstante, debido a la gran profusión de recursos posibles, faltan estudios científicos del probable intercambio de pulseras de caliza y de esquisto. En cambio, los brazaletes de jadeita y de concha se han estudiado más. Spondylus gaederopus es un molusco marino bivalvo que se cortaba para extraer anillos o brazaletes, cuentas y cilindros. La valva izquierda es delgada, por lo que se utilizaba para anillos finos. La derecha es en cambio más gruesa. Trabajos recientes llevados a cabo en Dímini, al Noroeste de Grecia, por el professor Hourmoziadis y la investigadora Akira Tsuneki (Tsuneki, 1989) muestran que durante el Neolítico final Dímini era uno de los centros en los que se manufacturaban anillos de spondylus. Se puede hablar de artesanía especializada. Desde la bahía de Volos, donde se obtenían las conchas, los brazaletes se comercializaban río arriba hasta Tesalia central y los Balcanes.

Los brazaletes hallados en Centroeuropa (periodos de Vinca y Linearbandkeramik) se localizaban principalmente en tumbas (Willms, 1982); los análisis de estroncio de tres cuentas halladas en Austria y Hungría(Shackleton y Elderfield, 1990) demuestran que se elaboraron con spondylus g. procedente del Mediterráneo, y no con materia fósil. También se utilizaban para brazaletes otros moluscos marinos, como el glycymeris (que se usó para la fabricación de artefactos tanto en Europa Central como en Córcega y España). Es sin duda necesario para los prehistoriadores saber más sobre el comercio y uso de los brazaletes del Neolítico europeo.

Con respecto a la cerámica, los estudios se centran en las variedades finas (Peacock, 1969; Malone, 1986). Recientemente se han llevado a cabo en el Suroeste francés sobre ejemplares del Neolítico inicial. Barnett (1990) ha demostrado que vasijas procedentes de tres zonas regionales (Montagne Noire, Valle del Tet y Valle del Hérault) eran transportadas al abrigo de Jean Cros. Este yacimiento ha mostrado una economía ambulante, por lo que es posible que algunas cerámicas fueran llevadas directamente por sus productores; pero parece probable que por lo menos ciertos recipientes decorados aparecidos en algunos yacimientos de la zona sean fruto del intercambio.

\subsection{Sistemas de comercio y abastecimiento}

El tercer método que se ha utilizado en el estudio del Neolítico europeo ha consistido en la investigación de sistemas de comercio y abastecimiento tanto a nivel local como regional. Volviendo al abrigo de Jean Cros, podemos decir que, además de importar cerámica, se traía sílex y piedra recorriendo distancias de diez a veinte kilómetros. Llegaron además conchas procedentes de la costa (Guilaine y otros, 1979). Un pequeño asentamiento de la Calabria, Piana de Curinga, utilizaba muchos recursos cercanos, con importación de obsidiana y otras materias pétreas (Ammerman, 1985).

A nivel regional, en el Sur de Francia existió un sistema complejo de comercio durante el Neolítico medio (Phillips, 1982). Desde el valle del Rhone se intercambiaba sílex por diferentes materiales, como por ejemplo variscita procedente de Can Tintorer, ecologita (jadeita) de Liguria y Piamonte (Ricq de Bouard y otros, 1990), así como obsidiana importada de Cerdeña o las Lípari (Williams Thorpe y otros, 1984). Este comercio de tan largo alcance puede deberse a especialistas (Binder y Perlès, 1990). También se intercambiaba cerámica decorada, bolitas de piedra, brazaletes, algunos tipos de hachas y molederas de arenisca; pero suponemos que estos intercambios eran más locales. Parece que el trueque se producía en grandes asentamientos de llanura, por ejemplo cerca de los ríos Garonne, Aude y Rhone (Phillips, 1982; Beeching y otros, 1991). 


\section{CONCLUSIONES}

La importancia del comercio para los grupos iniciales de agricultores reside en el hecho de que propicia contactos contínuos entre comunidades. Dichos vínculos fueron esenciales para el aprovisionamiento de artículos de subsistencia, materias primas, novias y novios (parejas), y para rebajar la posibilidad de conflictos sociales.

Durante el Neolítico final es posible que el prestigio -de un individuo o de toda una familia- haya jugado un papel importante. Los métodos científicos "prueban" la existencia de intercambios comerciales. Después de los análisis científicos, corresponde a los prehistoriadores explicar el papel de la producción, del intercambio y del uso de las materias primas dentro de la vida social en el Neolítico europeo.

Petrología

$\mathbf{M}$

$\mathbf{E}$

$\mathbf{T}$

$\mathbf{0}$

D

$\mathbf{0}$

S
Análisis isotópico

Elementos mayores, menores, traza

Análisis por activación de neutrones

\author{
Microscopía de barrido \\ electrónico \\ Análisis por microprueba \\ electrónica \\ Resonancia de espín \\ electrónica \\ Espectroscopía de plasma \\ inductivamente aclopado
}

\section{BIBLIOGRAFÍA}

AMMERMAN, A. J. (1985): Acconia Survey: Neolithic Italy and the Obsidian Trade. London. University of London Institute of Archaeology. Occasional Paper 10.

BARNETT, W. K. (1990): "Small-scale transport of early Neolithic pottery in the West Mediterranean", Antiquity 64, 245: 859-865.

BECK, C. W. y SHENNAN, S. (1991): Amber in Prehistoric Britain. Oxford. Oxbow Monograph 8.

BEECHING, A. (1991): "Sépultures, territoires et sociétés dans le Chasséen méridional. L'exemple du Bassin Rhodanéen", en BEECHING, A., BINDER, D., BLANCHET, J-C., CONSTANTIN, C., DUBOULOZ, J., MARTÍNEZ, F., MORDANT, D., THÉVENOT, J-P. y VAQUER, J. (eds.) Identité du Chasséen. Nemours. Mémoires du Musée de la Préhistoire de l'Ile de France 4: 37-41.

BINDER, D. y PERLES, C. (1990): "Stratégies de Gestion des Outillages Lithiques au Néolithique", Paléo 2: 257-283.

BOGUCKI, P. I. (1988): Forest Farmers \& Stockherders. Early Agriculture and its consequences in NorthCentral Europe. Cambridge: Cambridge University Press. 
BRADLEY, R. y EDWARDS, M. (1993): Interpreting the Axe Trade: Production and Exchange in neolithic Britain. Cambridge. Cambridge University Press.

BROOKS, I.P. (1989): “Debugging the system: the characterization of flint by micropalaeontology”, en BROOKS, I.P. y PHILLIPS, P. (eds.): Breaking the stony silence (British Archaeological Report 213): 53-71. Oxford.

BROTHWELL, D. y HIGGS, E. S. (eds.) (1963): Science and Archaeology. London: Thames y Hudson.

CASE, H. (1969): “Neolithic Explanations", Antiquity 43, 171:176-186.

CLOUGH, T. y CUMMINS, W. (eds.) (1979 y 1988) Stone Axe Studies 1 y 2. London. Council for British Archaeology Research Reports 23 y 67.

COLES, B. y COLES, J. (1991): Sweet Track to Glastonbury. London: Thames y Hudson.

COWELL, M. R. y BOWMAN, S. G. E. (1985): "Provenancing and dating of flint", en PHILLIPS, P. (ed.), The Archaeologist and the Laboratory. London. Council for British Archaeology Research Report 58: 36-40.

ENGELEN, F. H. G. (ed.) (1981): Deerde Internationale Symposium over Vuursteen/Third International Symposium on Flint. Heerlen. Nederlandse Geologische Vereniging.

FRANCAVIGLIA, V. y PIPERNO, M. (1987): “La répartition et la provenance de l'obsidienne archéologique de la Grotta dell' Uzzo et de Monte Cofano (Sicile)". Révue d'Archéometrie 11:31-39.

GUILAINE, J., GASCO, J., VAQUER, J. y BARBAZA, M. (1979): L'abri Jean Cros. Toulouse. Centre d'Anthropologie des Sociétés Rurales.

HARDING, T. G. (1967): Voyagers of the Vitiaz Straits. A Study of a New Guinea Trading System. Seattle. University of Washington Press. Monograph of the American Ethnological Society 44.

HUGHES, I. (1977): New Guinea Stone Axe Trade. The Geography and Ecology of Traffic in the Interior. Canberra. Department of Prehistory, Research School of Pacific Studies, ANU. Terra Australis 3.

JOHNSTONE, P. (1980): The Seacraft of Prehistory. London. Routledge y Kegan Paul.

MALONE, C. (1986): "Neolithic Exchange and Ritual Networks in the Central Mediterranean (5000-3000 b.c.)". The neolithic of Europe. The World Archaeological Congress, Southampton. Allen y Unwin.

MASSON, A. (1984): “Analyse pétrographique des silex utilisés par les néolithiques de l'Ile Corrège à Leucate, en GUILAINE, J., FREISES, A. y MONJARDIN, R. (eds.), Leucate-Corrège. Habitat Noyé du Néolithique Cardial. Sète. Musée Paul Valery: 59-71.

MAUSS, M. (1925): "Essai sur Le Don. Forme et raison de l'échange dans les sociétés archaiques", Année Sociologique, Paris, $2^{\mathrm{a}}$ serie 1: 30-186.

PEACOCK, D. (1969): "Neolithic pottery production in Cornwall", Antiquity 43: 145-149.

PERLES, C. (1990a): "L'outillage de la pierre taillée en Grèce: approvisionnement et exploitation des matières premières", Bulletin de Correspondance Hellénique. 114, 1: 1-42.

- (1990b): Les Industries Lithiques Taillées de Franchthi (Argolide, Grèce), Tome 2, en JACOBSEN, T. W. (ed.), Excavations at the Franchthi Cave, Greece, Fascicule 5. Bloomington e Indianapolis. Indiana University Press.

PHILLIPS, P. (1982): The Middle Neolithic in Southern France. Chasseen Farming and Culture Process. British Archaeological Report International Series 42.

— (1986): "Sardinian obsidian and neolithic exchange in the West Mediterranean", en BALMUTH, M. (ed.) Studies in Sardinian Archaeology II. Ann Arbor. University of Michigan Press: 203-209

RAMOS MILLÁN, A. (1991): "Immersion Exoscopic Analysis and sourcing subbetic flints", VI Symposium Internacional del Sílex: 261-263. Madrid.

RAMOS MUÑOZ, J. (e.p.): "Talleres líticos de la Prehistoria reciente en Cádiz". II Congreso Internacional "El Estrecho de Gibraltar" (Ceuta 1990): 203-227.

RENFREW, C.(1984): "Trade as action at a distance, en RENFREW, C. (ed.) Approaches to Social Archaelogy. Edinburgh. University Press: 86-134. 
RENFREW, C. y ASPINALL, A. (1990): “Aegen obsidian and Franchthi Cave, en PERLES, C. (1990b): 257270.

RICQ DE BOUARD, M., COMPAGNONI, R., DESMONS, J. y FEDELE, F., (1990): "Les roches alpines dans l'outillage poli néolithique de la France Méditerranéenne", Gallia Préhistoire 32: 125-149.

SHACKLETON, J. y ELDERFIELD, H. (1990): "Strontium isotope dating of the source of neolithic Spondylus shell artefacts", Antiquity 64: 312-315.

SIEVEKING, G. y NEWCOMER, M. (eds.) (1987): The Human Uses of Flint and Chert. Cambridge. Cambridge University Press.

TORRENCE, R. (1986): Production and Eschange of Stone Tools. Cambridge. Cambridge University Press.

TSUNEKI, A. (1989): “The manufacture of Spondylus shell objects at Neolithic Dimini, Greece". Orient 25: 1-21.

VALLESPI, E., RAMOS MUÑOZ, J., y CASTIÑEIRA, J. (1988): “Talleres líticos del Calcolítico y Bronce en la Sierra de Huelva y El Andevalo", Huelva en su Historia 2: 127-146. Huelva.

VAN ANDEL, T.H. y RUNNELS, C.N. (1988): "An Essay on the "Emergence of Civilization" in the Aegean World", Antiquity 62: 234-247

VILLALBA, M.J., BLASCO, A., EDO, M., ARENAS, J.A., y BANOLAS, L. (1989): “Mineria Neolítica. Can Tintorer, una aportación fundamental", Revista de Arqueología 96: 12-24

WILLIAMS THORPE, O.W., WARREN, S. E. y BARFIELD, L.H. (1979): "The sources and distributions of archaeological obsidian in Northern Italy", Prehistoria Alpina 15: 73-92.

WILLIAMS THORPE, O.W., WARREN, S. E. y COURTIN, J. (1984): “The distribution and sources of archaeological obsidian from Southern France", Journal of Archaeological Science 11: 135-146.

WILLIAMS THORPE, O.W., WARREN, S.E., y NANDRIS, J.G. (1984): "The distribution and provenance of archaeological obsidian in Central and Eastern Europe, Journal of Archaeological Science 11: 183-212.

WILLMS, C. (1982): Zwei Fundplätze der Michelsbergerkultur aus dem westlichen Münsterland, Münstersche Beiträge zu Ur- und Fruhgeschichte XII.

WOOLLEY, A.R. (1983): "Jade Axes and Other Artefacts", en KEMPE, D.R.C. y HARVEY, A.P. (eds.), The Petrology of Archaeological Artefacts: 256-276. Oxford. Clarendon Press. 